\title{
Efecto de la densidad de siembra sobre el crecimiento y supervivencia larval del pargo lunarejo Lutjanus guttatus
}

\author{
Effect of stocking density on survival and growth of larval spotted rose snapper \\ Lutjanus guttatus larvae
}

\section{Isabel Abdo-de la Parra ${ }^{1}$, L. Estela Rodríguez-Ibarra ${ }^{1}$, Fernando Campillo-Martínez ${ }^{1}$, Gabriela Velasco-Blanco ${ }^{1}$, Noemí García-Aguilar $\mathbf{N}^{1}$, Luis S. Álvarez-Lajonchère ${ }^{1}$ y Domenico Voltolina ${ }^{2}$}

\author{
${ }^{1}$ Centro de Investigación en Alimentación y Desarrollo, A.C., Av. Sábalo-Cerritos S/N, C. P. 82010 Mazatlán, Sinaloa, México \\ ${ }^{2}$ Laboratorio UAS-CIBNOR, Ap. Postal 1132, Mazatlán, Sinaloa, México \\ abdo@ciad.mx
}

\begin{abstract}
The effect on growth and survival of the initial stocking density (30, 20 and 10 eggs $\mathrm{L}^{-1}$ ) in larval rearing of spotted rose snapper (Lutjanus guttatus) was evaluated. The eggs were incubated in nine black fibreglass tanks with three replicates for treatment. Larvae were cultured in the same tanks using the green water technique. Larvae were harvested at 45 days post-hatch (DDE). Total length (LT), weight (PH) and
\end{abstract}

survival rate $(\mathrm{S})$ were determined. Initial stocking density did not affect growth and survival rates. Therefore, it is advisable to carry out larval culture of the spotted rose snapper using 30 eggs $\mathrm{L}^{-1}$ to maintain good growth levels.

Key words: Fish larvae, culture density, fish growth, larvae survival

\section{Introducción}

México presenta un excelente potencial para la acuicultura marina con sus casi $10.000 \mathrm{~km}$ de costa que incluye 1,6 millones de hectáreas de lagunas, bahías y estuarios; sin embargo, la maricultura ha estado restringida a la producción de camarón y a algunas especies de moluscos, principalmente ostiones. Aunque la producción de camarón ha aumentado cada año (FAO 2005), esta industria se ha desestabilizado en la última década por la incidencia de varias enfermedades. En la actualidad, el gobierno mexicano, los acuicultores y la academia están sumando esfuerzos en la expansión y diversificación de la maricultura.

El pargo lunarejo Lutjanus guttatus (Steindachner, 1869) es una de las especies de peces con potencial para su cultivo, esta es un importante recurso pesquero con un alto valor comercial en los mercados tanto nacionales como internacionales (Davis et al. 2000). Dentro de las investigaciones que se han realizado en el Centro de Investigación en Alimentación y Desarrollo, Unidad Mazatlán (CIAD) en los últimos años, han demostrado la factibilidad de su cultivo (García-Ortega et al. 2005', Ibarra \& Duncan 2007, Duncan et al. 2008, García-Ortega
2009, Ibarra-Castro \& Álvarez-Lajonchère 2009); sin embargo, una de las principales limitantes para llevar a cabo el cultivo a nivel comercial es la producción confiable y constante de juveniles (Álvarez-Lajonchère et al. 2007). Durante la larvicultura de peces marinos bajo condiciones controladas existen muchos factores que influyen sobre el crecimiento y la supervivencia; uno de ellos es la densidad de siembra de huevos o larvas, que puede afectar la calidad del agua, limitar el espacio y aumentar la agresividad de las larvas (Sakakura \& Tsukamoto 2002, Szkudlarek \& Zakés 2007). Por lo tanto, determinar los efectos de densidad así como la densidad óptima de siembra de huevos o larvas de las especies que se desean cultivar, es fundamental, para lograr la producción masiva de larvas de buena calidad a precios aceptables para los productores. Boza-Abarca et al. (2008) reportaron el cultivo larvario del pargo lunarejo a una densidad de siembra de 20 larvas $\mathrm{L}^{-1}$; en otras especies de pargo se han reportado densidades de siembra de 10 larvas L $L^{-1}$ (Watanabe et al. 1998, Leu et al. 2003). En otros estudios con peces marinos se han reportado densidades de siembra desde 1 a 200 huevos o larvas $\mathrm{L}^{-1}$, con resultados muy variables, dependiendo de la especie (Tucker 1998, Álvarez-González et al. 2001,

\footnotetext{
${ }^{1}$ García-Ortega A, I Abdo, N Duncan, E Rodríguez, G Velasco, B González, A Puello \& I Martínez. 2005. Larval rearing of rose spotted snapper Lutjanus guttatus under experimental conditions. En: Hendry CI, G van Stappen, M Wille \& P Sorgeloos (eds). Larvi’05. Fish and shellfish larviculture symposium, Ghent, European Aquaculture Society, Oostende, Belgium. Special Publication 36: 591
} 
Álvarez-Lajonchère \& Hernández-Molejón 2001, Benneti et al. 2008). Hasta la fecha no existen reportes sobre la densidad de siembra óptima para el cultivo larvario del pargo lunarejo, por lo que el objetivo del presente trabajo fue determinar el efecto de la densidad de siembra sobre el crecimiento y supervivencia de larvas de esta especie.

\section{Material y métodos}

\section{Obtención e incubación de huevos}

Los huevos fértiles se obtuvieron del lote de reproductores del laboratorio de reproducción de peces marinos del CIAD Unidad Mazatlán; las hembras fueron inducidas de acuerdo al protocolo propuesto por IbarraCastro \& Álvarez-Lajonchère (2009) con la hormona GnRHa ([D-Ala ${ }^{6}$ Pro $^{9}$ NEt]-GnRH) contenida en implantes de Ethylene-vinyl Acetate copolymer (EVAc) de liberación media (Zohar \& Mylonas 2001). Posterior a la inducción, se colocaron junto con 15 machos maduros naturalmente, en un tanque circular de fibra de vidrio de $18 \mathrm{~m}^{3}$ provisto de aireación, flujo continuo de agua marina y un colector de huevos forrado con malla de $500 \mu \mathrm{m}$. Los desoves empezaron $48 \mathrm{~h}$ después de la inducción y se seleccionaron los huevos que presentaban la mejor calidad, definida como el potencial de los huevos para producir larvas viables. Este potencial se evaluó de acuerdo a los criterios de Kjorsvik et al. (1990), seleccionándolos por su buena flotabilidad y por su coloración ámbar tenue a rosados.

El experimento se realizó en las instalaciones del criadero de la Unidad Mazatlán, en 9 tanques negros circulares de fibra de vidrio con una capacidad de $600 \mathrm{~L}$, equipados con aireación y suministros de agua continuos y regulables en forma independiente. El agua de mar se bombeó desde la playa Brujas y se filtró a través de filtros de arena con capacidad de retención de partículas de 16 $\mu \mathrm{m}$ de diámetro y por un sistema de irradiación UV de flujo continuo con una potencia mínima de emisión de $60 \mathrm{~mJ} \mathrm{~cm}^{-2}$.

En cada uno de tres tanques se sembraron 10 ó 20 ó 30 huevos fértiles $\mathrm{L}^{-1}$; los tratamientos fueron distribuidos al azar. Se incubaron en sistema estático, sin flujo de agua, solo con aireación. Para evaluar la tasa de eclosión se tomaron cuatro muestras de $1 \mathrm{~L}$ en diferentes partes de cada tanque y se contó el número de larvas vivas y muertas y el número de huevos no eclosionados de cada muestra. El porcentaje de eclosión (\% E) se calculó con la ecuación:

$$
\% \mathrm{E}=\frac{\text { Larvas vivas + larvas muertas }}{\begin{array}{c}
\text { Larvas }+ \text { larvas }+ \text { huevos no } \\
\text { vivas muertas eclosionados }
\end{array}} \times 100
$$

\section{Cultivo larvario}

Se llevó a cabo en los mismos tanques donde se incubaron los huevos, a una temperatura media de $31 \pm 0,5^{\circ} \mathrm{C}$ y a una salinidad de $35 \pm 1,0$ ups. Diariamente se registró la temperatura, la salinidad y se verificó que las concentraciones de $\mathrm{O}_{2}$ y de amonio disueltos permanecieran dentro de los límites aceptables (5,0 ppm y 0,05 ppm, respectivamente, Álvarez-Lajonchère \& Hernández-Molejón 2001). Cuando se notó que los valores de estas variables superaron los límites establecidos, el problema se resolvió incrementando el flujo de agua. Las larvas se cultivaron con luz continua durante los primeros días; desde los 16 días después de la eclosión (DDE), el fotoperiodo se redujo a 12:12 h luz-oscuridad. El filtro central de los tanques se cubrió con mallas de diferente abertura para evitar el escape del alimento vivo y las larvas permitiendo al mismo tiempo el recambio de agua. Hasta los 30 DDE se usó una malla de $100 \mu \mathrm{m}$ de abertura; 31 DDE y hasta la cosecha (45 DDE) se usó una malla de 500 ìm. Los filtros se cambiaron una vez al día para lavarlos y desinfectarlos. Entre los 2 hasta los 13 DDE se recambiaron diariamente aproximadamente $100 \mathrm{~L}$ de agua en cada tanque por medio de sifoneo del fondo. A partir del 14 DDE se inició el recambio por flujo contínuo con 0,2 vol día $^{-1}$ hasta el 16 DDE. De los 17 a los 19 DDE se recambiaron 0,5 vol día $^{-1}$. De los 20 a los 30 DDE se recambió un volumen de 0,8 a 1,0 día $\mathrm{a}^{-1}$. El recambio aumentó de 1 a 3 vol día ${ }^{-1}$ desde los 31 a los 45 DDE.

Las larvas se cultivaron mediante la técnica de agua verde, a partir de 1 y hasta los 13 DDE se añadió a cada tanque una mezcla de 500,000 células $\mathrm{mL}^{-1}$ de Nannochloropsis oculata y 50,000 células $\mathrm{mL}^{-1}$ de Isochrysis sp. Del 2 hasta el 6 DDE se añadieron a cada tanque 10 rotíferos $\mathrm{mL}^{-1}$ (Brachionus rotundiformis), previamente enriquecidos con microalgas. A partir de los 7 hasta los 15 DDE se aumentó a 20 rotíferos $\mathrm{mL}^{-1}$. A partir de los 2 DDE se añadió también una concentración de 0,5 copépodos $\mathrm{mL}^{-1}$ (Tisbe monozota y Pseudodiaptomus euryhalinus), que se mantuvo constante hasta los 15 DDE. Entre los 16 y 30 DDE la cantidad de rotíferos se redujo a $10 \mathrm{~mL}^{-1}$ y se añadieron 0,5 nauplios de Artemia $\mathrm{mL}^{-1}$, que se incrementaron gradualmente hasta $4 \mathrm{~mL}^{-1}$. El alimento vivo presente en cada tanque se contó diariamente para ajustarlo a la cantidad requerida. El cambio a alimento inerte se realizó a partir de los $30 \mathrm{DDE}$, con una dieta comercial microparticulada (Lansy 2/4, 4/6, 5/8 8/12, INVE Aquaculture México, Mazatlán, Sinaloa, México) para terminar a los 38 DDE, reduciendo gradualmente el número de nauplios de Artemia $\mathrm{mL}^{-1}$, hasta sustituirlos completamente por la dieta artificial. El protocolo de alimentación y manejo del agua se puede observar en la Fig. 1. 


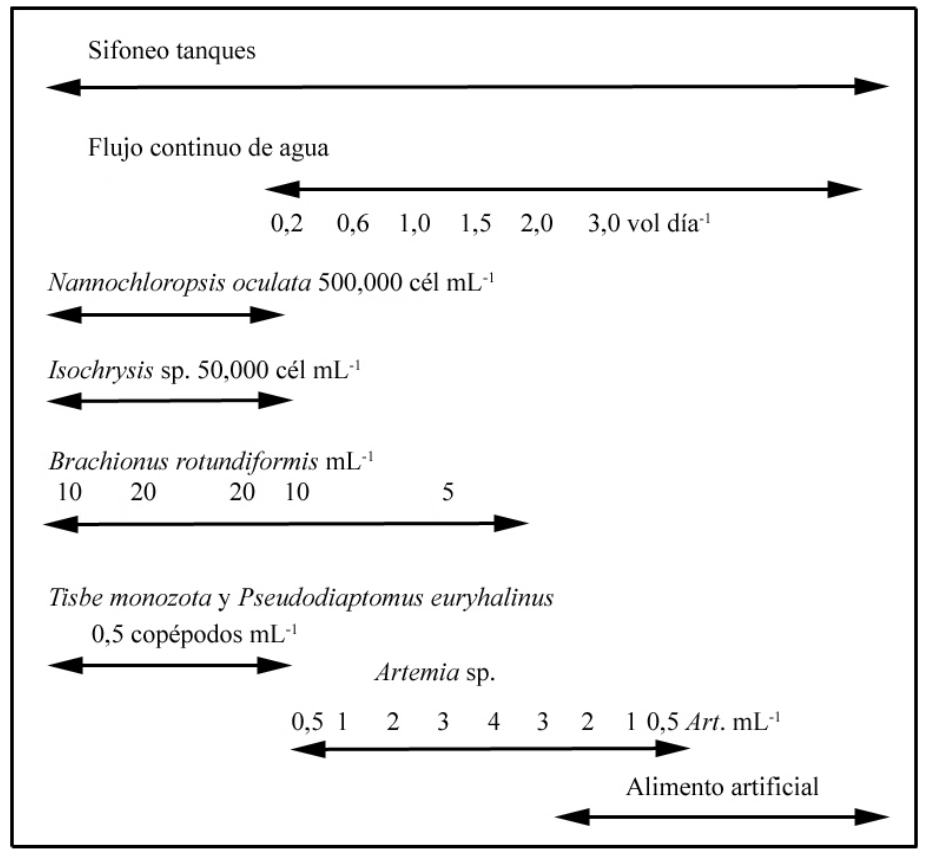

$\begin{array}{llllllllllllllllllllllll}0 & 2 & 4 & 6 & 8 & 10 & 12 & 14 & 16 & 18 & 20 & 22 & 24 & 26 & 28 & 30 & 32 & 34 & 36 & 38 & 40 & 42 & 44 & 46\end{array}$

Días después de la eclosión (DDE)

\section{Figura 1}

Procedimiento de alimentación y manejo de la calidad de agua utilizado en el cultivo larvario del pargo lunarejo L. guttatus

Feeding and water management protocol during larval rearing of spotted rose snapper L. guttatus

\section{Toma de muestras}

Cada cinco días se recolectaron al azar, 20 larvas de cada tanque, para determinar su longitud total (LT) en milímetros; se anestesiaron individualmente en $500 \mathrm{~mL}$ de agua de mar con $100 \mu$ l de 2-fenoxietanol (Sigma Aldrich®, P1126, Toluca, Edo. México) y con una pipeta Pasteur se colocaron en una caja Petri de cristal de $60 \mathrm{x}$ $15 \mathrm{~mm}$; se observaron a través de un microscopioestereoscopio y se midieron con un vernier digital (precisión 0,001 mm). A partir de los 15 DDE y hasta el momento de la cosecha, se obtuvo el peso húmedo individual (PH) por medio de una balanza analítica con precisión de 0,001 g. El porcentaje de supervivencia (\% S) se evaluó al final del experimento, contando todas las larvas presentes en cada tanque al momento de la cosecha (45 DDE), aplicando la ecuación:

$$
\% \mathrm{~S}=\left(\mathrm{N}^{\circ} \text { final larvas } / \mathrm{N}^{\circ} \text { inicial larvas }\right) \times 100
$$

El número inicial de larvas se determinó tomando en cuenta el número de huevos sembrados y el porcentaje de eclosión (Tabla 1).

\section{Análisis estadístico}

Los datos de porcentaje de eclosión y de supervivencia se transformaron a arcoseno para su posterior análisis. A todos los resultados se les determinó la normalidad de su distribución (prueba de Bartlet), así como la homocedasticidad de la varianza (prueba de Levene). Los datos fueron normales y se analizaron mediante un análisis de varianza de una vía $(P<0,05)$ (Zar 1996). Para describir el crecimiento, se aplicaron los modelos de regresión lineal y exponencial y se compararon con una prueba de ANOVA.

\section{Resultados y discusión}

El inicio de la eclosión se registró a partir de las 20 h después de la fertilización. El porcentaje medio de eclosión varió entre 82,3 y 93,1\%, sin que se registraran diferencias significativas entre los tres tratamientos $(P=$ 0,17 ) (Tabla 1); estos resultados son similares a los obtenidos en investigaciones previas con la misma especie por Ibarra \& Duncan (2007) y Duncan et al. 
Tabla 1

Porcentaje promedio ( \pm desviación estándar) de eclosión, longitud total (LT), peso húmedo (PH) y supervivencia (\% S) de larvas de $L$. guttatus al final del cultivo larvario (45 DDE) a tres densidades de siembra

Hatching rate ( \pm standard deviation), total length (LT), weight $(\mathrm{PH})$ and survival (\%S) obtained at 45 DDE of L. guttatus larvae at three stocking densities

\begin{tabular}{lcrccc}
\hline $\begin{array}{c}\text { Densidad de } \\
\text { siembra }\end{array}$ & $\begin{array}{c}\text { \% de } \\
\text { eclosión }\end{array}$ & Larvas L $^{-1}$ & LT $(\mathrm{mm})$ & PH $(\mathrm{g})$ & $\% \mathrm{~S}$ \\
\hline 30 Huevos L & & & & \\
\hline 20 Huevos L & $91,1 \pm 4,7$ & $27,3 \pm 1,2$ & $45,89 \pm 5,46$ & $1,41 \pm 0,45$ & $1,44 \pm 0,89$ \\
10 Huevos L & $82,3 \pm 8,2$ & $16,4 \pm 1,5$ & $48,23 \pm 8,40$ & $1,61 \pm 0,87$ & $1,45 \pm 0,14$ \\
& $93,1 \pm 6,0$ & $9,3 \pm 0,6$ & $42,70 \pm 12,00$ & $1,35 \pm 0,95$ & $2,82 \pm 0,19$ \\
\hline
\end{tabular}

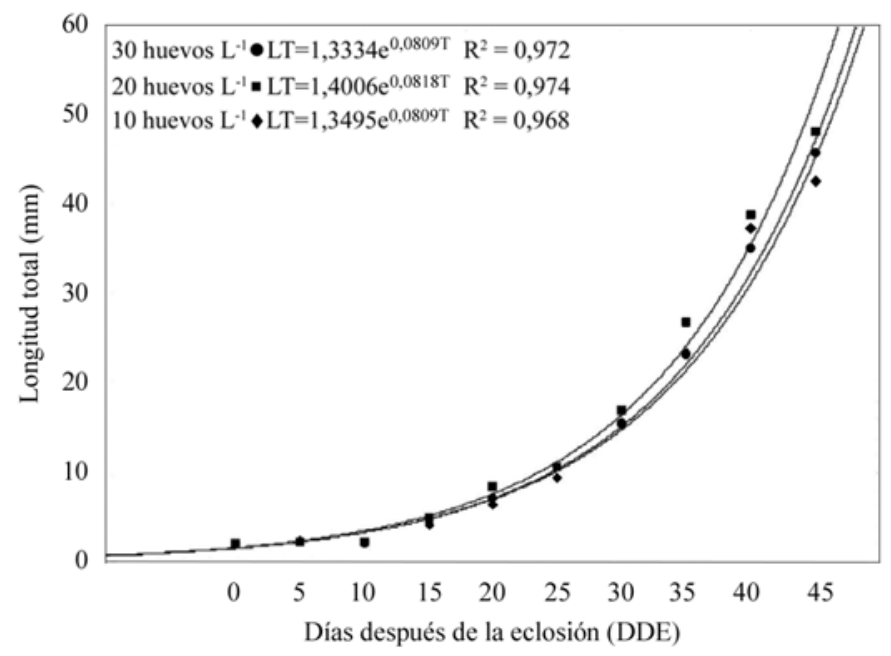

Figura 2

Curvas de crecimiento de larvas del pargo L. guttatus basadas en la LT durante 45 DDE en diferentes densidades de siembra

Growth tendencies based on TL of spotted rose snapper L. guttatus larvae at the different stocking densities until 45 DDE

(2008) quienes reportaron porcentajes de eclosión entre 76 a $97 \%$. Bourque \& Phelps (2007) indicaron que en el pargo rojo (L. campechanus) no hay una relación entre el porcentaje de eclosión y la densidad de incubación. La LT promedio inicial de las larvas en todos los tratamientos fue 2,18 \pm 0,15 mm; Ibarra-Castro (2008) y Boza-Abarca et al. (2008) reportaron para la misma especie una LT inicial de 2,1 a 2,38 mm, para otras especies de pargo como L. campechanus se ha reportado una LT inicial de 2,2 \pm 0,01 mm (Papanikos et al. 2003), por lo cual inferimos que las densidades de siembra utilizadas en este estudio no interfieren con el proceso del desarrollo embrionario y eficiencia de la eclosión. A los 2 DDE se observó la abertura del poro anal y de la boca y a los 3 DDE inició la alimentación exógena. En la Tabla 1 se aprecian los resultados obtenidos al final del cultivo larvario. Se obtuvo una LT final de 42,7 a 48,3 $\mathrm{mm}$ y no se observaron diferencias significativas entre los tres tratamientos $(P=0,531)$. Se confirmó que el crecimiento es de tipo exponencial con coeficientes de determinación $\left(\mathrm{R}^{2}\right)$ cercanos o superiores a 0,97 (Fig. 2). El PH final de las larvas no presentó diferencias significativas entre los tratamientos $(P=0,645)$, varió entre 1,35 a 1,61 g. Boza-Abarca et al. (2008) llevaron a cabo el cultivo de L. guttatus con una densidad inicial de 20 huevos $\mathrm{L}^{-1}$ y reportaron crecimientos similares a los obtenidos en el presente estudio. En cuanto a la supervivencia en el presente trabajo, tampoco se detectaron diferencias significativas entre los tratamientos ( $P=0,13)$, variando entre 1,5 y $2,8 \%$. Actualmente no hay otros reportes de supervivencia a los $45 \mathrm{DDE}$ para esta especie; sin embargo Boza-Abarca et al. (2008) 
mencionan la supervivencia a los 62 DDE la cual fue de 1,5\%; Turano et al. (2000) reportan una supervivencia de 2,8\% a los 45 DDE para el pargo de cola amarilla (Ocyurus chrysurus) y para el pargo criollo (Lutjanus annalis) a una densidad de siembra de 10,5 huevos $\mathrm{L}^{-1}$ se reportó una supervivencia de $14,3 \%$ a los 38 DDE (Watanabe et al. 1998).

La densidad de siembra puede afectar significativamente el crecimiento y la supervivencia en los cultivos de larvas de peces marinos (Houde 1975, Tucker 1998, Montero et al. 1999); los resultados de varios estudios en otras especies de peces marinos han demostrado que la densidad de siembra afecta tanto el crecimiento como la supervivencia. En la cobia (Rachycentron canadum), el efecto de la densidad de siembra en el crecimiento y supervivencia se observa desde densidades mayores a 10 larvas L ${ }^{-1}$ (Hitzfelderg et al. 2006, Benneti et al. 2008); en el lenguado (Paralichthys dentatus) el crecimiento de las larvas se afecta también a densidades mayores a 10 larvas $\mathrm{L}^{-1}$, ya sea en condiciones de laboratorio o a escala comercial (King et al. 2000). Para el cultivo larvario intensivo del dentón común (Dentex dentex) se sugieren densidades de siembra de 10 a 40 larvas $\mathrm{L}^{-1}$ (Giménez \& Estévez 2008). Sin embargo, en otras especies como la cabrilla arenera (Paralabrax maculatofasciatus) y la lubina (Dicentrarchus labrax) se ha reportado que soportan densidades de siembra mayores a 50 larvas $\mathrm{L}^{-1}$ (Álvarez-González et al. 2001, Conides \& Glamuzina 2001). En el presente estudio, las tres diferentes densidades de siembra que se utilizaron no afectaron el crecimiento y aunque los porcentajes de supervivencia obtenidos en los tres tratamientos no presentaron diferencias, resultaron inferiores a los deseables para una producción a nivel comercial (Tucker 1998, ÁlvarezLajonchère \& Hernández-Molejón 2001) por lo que es necesario realizar estudios con densidades más altas para observar los efectos que pueda tener la densidad de siembra sobre el cultivo larvario.

En base a los resultados del presente estudio, se asume que el cultivo larvario del pargo lunarejo puede llevarse a cabo con una densidad inicial de 30 huevos $\mathrm{L}^{-1}$ sin afectar la eficiencia de la eclosión y crecimiento de las larvas.

\section{Agradecimientos}

Los autores agradecen la asistencia técnica de A. Ibarra, M. Cruz, J. Huerta y a la bióloga V.P. Domínguez-Jiménez por los análisis de calidad de agua de los cultivos y a V. Williams de Calvario por la traducción del resumen y revisión del texto. Este trabajo fue financiado por el
Fondo Mixto Sinaloa (FOMIX) SIN-2006 C01- 37105 dirigido por M.I. Abdo de la Parra.

\section{Literatura citada}

Álvarez-González C, S Ortíz-Galindo, S Dumas, E MartínezDomínguez, D Hernández-Ceballos, T Grayeb-del Alamo, M Moreno-Legorreta \& R Peña-Martínez. 2001. Effect of stocking density on the growth and survival of spotted sand bass Paralabrax maculatofasciutus larvae in a closed recirculating system. Journal of the World Aquaculture Society 32(1): 130-137.

Álvarez-Lajonchère L \& OG Hernández-Molejón. 2001. Producción de juveniles de peces estuarinos para un Centro en América Latina y el Caribe: diseño, operación y tecnologías, 424 pp. World Aquaculture Society, Baton Rouge.

Álvarez-Lajonchere LS, MA Reina-Cañez, MA CamachoHernández \& S Kraul. 2007. Design of a pilot-scale tropical marine finfish hatchery for a research center at Mazatlán, México. Aquacultural Engineering 36: 81-96.

Benetti DD, B Sardenberg, A Welch, R Hoenig, MR Orhun \& I Zink. 2008. Intensive larval husbandry and fingerling production of cobia Rachycentron canadum. Aquaculture 281(1-4): 22-27.

Bourque BD \& RP Phelps. 2007. Induced spawning and egg quality evaluation of red snapper, Lutjanus campechanus. Journal of the World Aquaculture Society 38(2): 208-217.

Boza-Abarca J, C Calvo-Vargas, N Solis-Ortiz \& J Komen. 2008. Desove inducido y crecimiento del pargo manchado Lutjanus guttatus, en la Estación de Biología Marina de Puntarenas, Costa Rica. Ciencias Marinas 34(2): 239-252.

Conides AJ \& B Glamuzina. 2001. Study on the effects of rearing density, temperature and salinity on hatching performance of the European sea bass, Dicentrarchus labrax (Linnaeus, 1758). Aquaculture International 9: 217224.

Davis DA, KL Bootes \& CR Arnold. 2000. Snapper (Family Lutjanidae) culture. En: Stickney RR (ed). Encyclopedia of aquaculture, pp. 884-889. John Wiley \& Sons, New York.

Duncan NJ, L Ibarra-Castro \& R Álvarez-Villaseñor. 2008. Effect of the dusk photoperiod from light to dark on the incubation period of eggs of the spotted rose snapper, Lutjanus guttatus (Steindachner). Aquaculture Research 39: 422-433.

FAO. 2005. FISHSTAT Plus (Version 2.3) FAO, Roma. [CD$\mathrm{ROM}]$.

García-Ortega A. 2009. Nutrition and feeding research in the spotted rose snapper (Lutjanus guttatus) and bullseye puffer (Sphoeroides annulatus), new species for marine aquaculture. Fish Physiology and Biochemistry 35(1): 6980 . 
Giménez G \& A Estévez. 2008. Effect of larval and prey density, prey dose and light conditions on first feeding common dentex (Dentex dentex L.) larvae. Aquaculture Research 9: 77-84.

Hitzfelderg GM, J Holt, J Fox \& D McKee. 2006. The effect of rearing density on growth and survival of cobia, Rachycentron canadum, larvae in a closed recirculating aquaculture system. Journal of the World Aquaculture Society 37(2): 204-209.

Houde ED. 1975. Effects of stocking density and food density on survival, growth and yield of laboratory-reared larvae of sea bream Archosargus rhomboidalis (L.) (Sparidae). Journal of Fish Biology 7: 115-129.

Ibarra-Castro L. 2008. Efecto de variables exógenas y endógenas sobre la maduración sexual, desove y producción de larvas de pargo lunarejo (Lutjanus guttatus). Tesis de Doctorado, Centro de Investigación en Alimentación y Desarrollo, Mazatlán, 168 pp.

Ibarra-Castro L \& L Álvarez-Lajonchère. 2009. Improved induced-spawning protocol for the spotted rose snapper (Lutjanus guttatus) The Israeli Journal of AquacultureBamidgeh 61(2): 121-133.

Ibarra-Castro L \& N Duncan. 2007. GnRHa-induced spawning of wild-caught spotted rose snapper Lutjanus guttatus. Aquaculture 272: 737-746.

King NJ, WH Howell, M Huber \& DA Bengtson. 2000. Effects of larval stocking density on laboratory-scale and commercial-scale production of summer flounder Paralichthys dentatus. Journal of the World Aquaculture Society 31(3): 436-445.

Kjorsvik E, A Mangor-Jensen \& I Holmefjord. 1990. Eggs quality in fish. Advance in Marine Biology 26: 71-11.

Leu MY, Chen IH \& LS Fang. 2003. Natural spawning and rearing of mangrove red snapper, Lutjanus argentimaculatus, larvae in captivity. The Israeli Journal of AquacultureBamidgeh 55: 22-30.

Montero D, MS Izquierdo, L Tort, L Robaina \& JM Vergara. 1999. High stocking density produces crowding stress altering some physiological and biochemical parameters in gilthead seabream, Sparus aurata, juveniles. Fish Physiology and Biochemistry 20: 53-60.

Papanikos N, RP Phelps, K Williams, A Ferry \& D Maus. 2003. Egg and larval quality of natural and induced spawns of red snapper, Lutjanus campechanus. Fish Physiology and Biochemistry 28: 487-488.

Sakakura Y \& K Tsukamoto. 2002. Onset and development of aggressive behaviour in the early life stage of japanese flounder. Fish Science 68: 854-861.

Szkudlarek M \& Z Zakés. 2007. Effect of stocking density on survival and growth performance of pikeperch, Sander lucioperca larvae under controlled conditions. Aquaculture International 15: 67-81.

Tucker JW. 1998. Marine fish culture, 750 pp. Kluwer Academic Publishers, Boston.

Turano M, DA Davis \& CR Arnold. 2000. Observations and techniques for maturation, spawning and larval rearing of the yellow snapper Ocyurus chrysurus. Journal of the World Aquaculture 31(1): 59-68.

Watanabe WO, EF Ellis, SC Ellis, J Chávez \& C Manfredi. 1998. Artificial propagation of mutton snapper Lutjanus analis, a new candidate marine fish species for aquaculture. Journal of the World Aquaculture Society 29(2): 176-187.

Zar JH. 1996. Biostatistical analysis, 662 pp. Prentice-Hall, Englewood Cliffs.

Zohar Y \& C Mylonas. 2001. Endocrine manipulation of spawning in cultured fish: from hormones to genes. Aquaculture 197: 99-136.

Recibido el 11 de junio de 2009 y aceptado el 09 de noviembre de 2009 